\title{
Uso da monitoração ambulatorial da pressão arterial nos distúrbios hipertensivos gestacionais
}

\author{
Use of ambulatory blood pressure monitoring in the gestational \\ hypertensive disorders
}

\author{
Pâmela Fernanda Prado ${ }^{1}$, Stael Silvana Bagno Eleutério da Silva² \\ ${ }^{1}$ Centro Universitário Adventista de São Paulo (UNASP) - São Paulo (SP), Brasil. \\ ${ }^{2}$ Faculdade de Ciências Sociais e Agrárias de Itapeva (FAIT) - Itapeva (SP), Brasil.
}

DOI: http://dx.doi.org/10.7322/abcshs.v42i2.1011

\begin{abstract}
RESUMO
A gravidez é um fenômeno fisiológico, mas os danos gestacionais à saúde materna e fetal podem ocorrer durante o seu progresso. Os transtornos hipertensivos gestacionais, considerados a principal causa de óbitos obstétricos diretos, são responsáveis por altas taxas de morbidade e mortalidade materna e perinatal, o que os torna um grande problema de saúde pública. Sabe-se que há diferenças significativas entre a medida casual da pressão arterial em nível domiciliar e a obtida em nível ambulatorial e/ou hospitalar. Por isso, o uso da monitoração ambulatorial da pressão arterial (MAPA) é uma importante estratégia utilizada na confirmação do diagnóstico e no controle da hipertensão, pois permite a avaliação precisa da pressão arterial através de um registro sistematizado e eficiente. As pesquisas científicas confirmam a importância do uso da MAPA em relação aos transtornos hipertensivos, destacando, principalmente, sua correlação com os valores obtidos quando comparados à medida casual e à automedida residencial da pressão. Nessas circunstâncias, concluiu-se que a MAPA é uma ferramenta vantajosa no controle da pressão e para os cuidados com mulheres grávidas. A assistência prestada pelos enfermeiros obstétricos com o uso da MAPA é relevante porque minimiza a possibilidade de morte fetal e de morte materna por hipertensão, possibilitando planos de atendimento com base em níveis de pressão mais confiáveis do que a medida casual.
\end{abstract}

Palavras-chave: gravidez; hipertensão; monitorização ambulatorial da pressão arterial.

\begin{abstract}
Pregnancy is a physiological phenomenon, but gestational harm to maternal and fetal health may occur during its progress. Gestational hypertensive disorders are responsible for high rates of maternal and perinatal morbidity and mortality, and are considered the leading cause of direct obstetric deaths, representing a major public health issue. It is known that there are significant differences between the casual blood pressure measured at home and that obtained at the outpatient and/or hospital levels. Therefore, the use of ambulatory blood pressure monitoring (ABPM) is an important strategy used in the prevention of hypertension, because it allows the accurate evaluation of blood pressure through a systematized and efficient registry in the diagnosis and control of the disease. Scientific research confirms the importance of ABPM in relation to hypertensive disorders, mainly highlighting its correlation with the values obtained when compared to the casual measure and residential pressure measurement. Under these circumstances, this study concluded that ABPM is an advantageous tool in the control of blood pressure and care for pregnant women. The assistance provided by obstetrician nurses with the use of ABPM is relevant because it minimizes the possibility of fetal and maternal death by hypertension, enabling plans of care based on pressure levels more reliable than the casual measure.
\end{abstract}

Keywords: pregnancy; hypertension; blood pressure monitoring, ambulatory. 


\section{INTRODUÇÃO}

A gestação é um fenômeno fisiológico. No entanto, em sua evolução podem ocorrer agravos que colocam em risco a saúde materna e fetal. Dentre as doenças que acometem a mulher no período gravídico, a hipertensão induzida pela gravidez é uma das patologias que ocasionam graves eventos para o binômio mãe-filho ${ }^{1}$

Dois distúrbios hipertensivos estão entre os responsáveis pela pressão alta na gestante: a hipertensão crônica e a hipertensão induzida pela gravidez. Sobrepondo-se à hipertensão, encontram-se a pré-eclâmpsia e a eclâmpsia, responsáveis por taxas elevadas de morbimortalidade materna e perinatal ${ }^{2-4}$ e consideradas as principais causas de mortes obstétricas diretas. Com isso, as síndromes hipertensivas na gestação tornaram-se um grave problema de saúde pública ${ }^{5}$.

Em cerca de $10 \%$ das gravidezes de primíparas normotensas há presença da hipertensão gestacional, que consiste em níveis pressóricos elevados após a vigésima semana de gestação, ausentando-se até o décimo dia após o parto (hipertensão transitória) ${ }^{6}$.

Diversos órgãos da gestante são acometidos pela hipertensão arterial, incluindo, além dos órgãos-alvos, os olhos, rins, coração e cérebro, o fígado. Há também restrição do fluxo placentário devido ao estreitamento de vasos deciduais pelo surgimento da aterose. As complicações mais frequentes para o concepto associadas à patologia incluem: redução do crescimento fetal intrauterino; prematuridade; baixo peso do bebê ao nascer; e mortalidade fetal e neonatal?

Compete aos enfermeiros obstetras, seja no nível primário ou qualquer outro nível do cuidado com gestantes, a realização do pré-natal com ênfase na identificação de situações que agravam a saúde e, consequentemente, a realização de planos de cuidados individuais, tendo em vista o controle da evolução da patologia ${ }^{6}$. A assistência possui extrema relevância na prevenção da morbimortalidade materna e perinatal, visto que a pressão alta na gravidez, até então, não pode ser evitada, enquanto o óbito materno pode ser impedido na maioria das vezes ${ }^{1}$.

Considerando que a única forma de diagnosticar e controlar a hipertensão arterial é aferindo a pressão arterial (PA), sabendo-se que há significativas diferenças entre a medida casual (valores obtidos por medidas no consultório) e a medida no domicílio, os métodos que se utilizam de aparelhos automáticos afastam diversos fatores que interferem nessa medida. A monitorização ambulatorial da pressão arterial (MAPA) é um dos métodos que possibilita o armazenamento de um grande número de medidas, além de afastar a influência do consultório e o efeito do avental branco ${ }^{8}$.

Nos últimos anos, a MAPA vem se tornando uma ferramenta de extrema eficácia na clínica obstétrica, tanto em gestantes hipertensas quanto em normotensas com perfil clínico-epidemiológico para desenvolver distúrbio hipertensivo na gestação\%.

O uso da MAPA é uma importante estratégia para prevenção de agravos provocados pela hipertensão, pois permite a avaliação da PA por meio de um registro prolongado, ininterrupto e sistematizado, sendo o método mais eficiente e preciso no diagnóstico e controle da doença, além de permitir prever alguns eventos cardiovasculares ${ }^{10}$.

Diante da problemática e das justificativas citadas, esta pesquisa teve como objetivo descrever o uso da MAPA como estratégia no diagnóstico da hipertensão e no controle e assistência à gestante hipertensa.

\section{MÉTODOS}

A pesquisa bibliográfica teve início em novembro de 2015 e término em maio de 2016.

Para escolha das palavras-chave foram utilizados termos indexados junto aos Descritores em Ciências da Saúde (DeCS). Considerase esta uma pesquisa descritiva, pois busca descrever as características do que será estudado, por meio de levantamento bibliográfico relacionado à temática hipertensão arterial na gestação, bem como a utilização da MAPA no diagnóstico e controle da doença ${ }^{11}$.

$\mathrm{Na}$ pesquisa descritiva, os fatos observados são registrados e descritos pelo pesquisador sem que o mesmo interfira; essa pesquisa analisa, organiza, observa e registra os dados sem que sejam manipulados. No que se refere à pesquisa exploratória, os autores descrevem que esta tem como objetivo oferecer mais informações referentes ao assunto investigado, ou seja, possibilita o esclarecimento de um determinado tema pouco explorado ${ }^{12}$.

Para elaboração deste trabalho, as buscas foram realizadas em publicações científicas indexadas em bases de dados nacionais online, tais como Biblioteca Virtual de Saúde (BVS), Medical Literature Analysis and Retrieval System Online (MEDLINE) Scientific Eletronic Library Online (SciELO), além do PubMed. Os descritores utilizados foram: hipertensão gestacional / gestational hypertension; e monitorização ambulatorial da pressão arterial (MAPA) / ambulatory blood pressure monitoring (ABPM).

Um dos fatores limitadores desta pesquisa foi a dificuldade de acesso às publicações, considerando a temática conjunta (MAPA - gestação).

A pesquisa pretende fornecer informações pertinentes sobre o auxílio da MAPA no contexto da assistência à gestante com distúrbio hipertensivo, por meio de pesquisas bibliográficas, considerando autores renomados nacionais e internacionais nessa temática.

\section{RESULTADOS}

É claramente demonstrado em estudos que as medidas da PA realizadas no ambiente domiciliar preveem melhor os danos cardiovasculares e em órgãos-alvo do que as medidas convencionais de consultório. Dessa maneira, recomendações atuais sugerem a monitorização da PA no âmbito familiar, apresentando como papel primordial o diagnóstico preciso da hipertensão, o ajuste da terapêutica medicamentosa e o seguimento dos hipertensos ${ }^{13}$.

A MAPA é um método preciso por fornecer informações claras dos níveis pressóricos durante um longo período, não requisitando treinamento ou habilidade profissional para realização da 
medida da pressão. A sua utilização também não está sujeita a erros devido aos fatores comportamentais do paciente. Para o tratamento anti-hipertensivo, é importante esclarecer que, com sua utilização, é possível ter conhecimento da eficácia dos fármacos nas 24 horas anteriores ao uso, contribuindo para aperfeiçoamento do tratamento e, desse modo, reduzindo os custos ${ }^{14}$.

As diferenças obtidas entre a PA no domicílio e em consultório não são justificadas apenas pela rotina; acredita-se que fatores psicológicos devam estar envolvidos. Pacientes que apresentam diferenças na medida casual de consultório em comparação com as medidas domiciliares são erroneamente classificados como hipertensos (hipertensão do avental branco). Nas gestantes, a hipertensão do avental branco ocorre em 3,2 e 4,2\% dos casos, quando são avaliadas, respectivamente, as pressões sistólica e diastólica ${ }^{15,16}$.

Por meio da MAPA profissionais qualificados avaliaram o ritmo circadiano da PA de gestantes normotensas, e concluíram que essa monitorização pode ser utilizada para o diagnóstico da hipertensão arterial na gestação. Essa tem sido sugerida como a abordagem mais precisa para superar a baixa sensibilidade e especificidade das medidas casuais na gravide ${ }^{17,18}$.

A utilização da MAPA em gestantes pode auxiliar no diagnóstico precoce da pré-eclâmpsia, e quanto mais precocemente for feito o diagnóstico dessa condição clínica, em conjunto com intervenção em momento adequado, maiores são as possibilidades de haver uma gestação sem complicações, tanto materna quanto para o concepto. Dessa maneira, é possível afirmar que a MAPA é um instrumento vantajoso e muito importante de ser usado como estratégia na assistência das gestantes com distúrbio hipertensivo ${ }^{1,19}$.
As orientações para seu uso podem representar, futuramente, uma mudança no padrão de mensuração e controle da PA, uma vez que a clientela passará a ter uma atribuição mais ativa e importante referente ao conhecimento e acompanhamento dos níveis pressóricos e, dessa forma, interferindo positivamente na adesão ao tratamento e no controle da PA, reduzindo, assim, os malefícios ${ }^{20}$.

\section{CONCLUSÃO}

Os autores analisados confirmam a importância da MAPA como estratégia no controle e na assistência à gestante hipertensa, destacando principalmente a fidedignidade dos valores obtidos pela MAPA quando comparados aos outros métodos de medida da PA; a possibilidade de um tratamento mais preciso baseado nos níveis da PA mais exatos; a possibilidade de verificar medidas durante o sono, aumentando a confiabilidade dessas medidas; e o diagnóstico mais acurado da hipertensão arterial, favorecendo o adequado tratamento da doença.

Tanto para o diagnóstico quanto para o tratamento da hipertensão arterial, o uso da MAPA é um incremento fundamental para diminuir os agravos à saúde das gestantes hipertensas, pois permite uma excelente análise dos valores da pressão e, consequentemente, a correta interpretação e implementação da terapêutica, sendo capaz de permitir uma excelente análise de dados e, assim, o diagnóstico preciso.

As autoras dessa pesquisa concluem que na prática clínica a MAPA deveria fazer parte do diagnóstico e tratamento da hipertensão arterial na gestação em vista da possibilidade da redução de seus agravos à saúde do binômio mãe-filho.

\section{REFERÊNCIAS}

1. Chaim SRP, Oliveira SMJV, Kimura AF. Hipertensão arterial na gestação e condições neonatais ao nascimento. Acta Paul Enferm. 2008;21(1):53-8.

http://dx.doi.org/10.1590/S0103-21002008000100008

2. Sociedade Brasileira de Cardiologia. Sociedade Brasileira de Hipertensão. Sociedade Brasileira de Nefrologia. VI Diretrizes Brasileiras de Hipertensão Arterial. Arq Bras Cardiol. 2010;95(1 sup. 1):1-51.

http://dx.doi.org/10.1590/S0066-782X2010001700001

3. Brasil. Ministério da Saúde. Secretaria de Atenção à Saúde. Departamento de Ações Programáticas Estratégicas. Gestação de alto risco: manual técnico. 5ed. Brasília: Ministério da Saúde; 2012.

4. American College of Obstetricians and Gynecologists. Hypertension in pregnancy. Disponível em: http://www.acog. org/Resources-And-Publications/Task-Force-and-Work-GroupReports/Hypertension-in-Pregnancy. Acesso em: 22 fev. 2017.

5. Soares NS, Coutinho RFC, Queiroz RD, Sousa SPO, Sousa Nétto OB. Observatório epidemiológico: governo treina gestores municipais para reduzir mortalidade materna. 2010. Disponível em: http://www.ceut. com.br/observatorio/edicao\%2020.pdf. Acesso em: 04 dez. 2015.
6. Goncalves R, Fernandes RAQ, Sobral DH. Prevalência da doença hipertensiva específica da gestação em hospital público de São Paulo. Rev Bras Enferm. 2005;58(1):61-4 http://dx.doi.org/10.1590/S0034-71672005000100011

7. Pascoal IF. Hipertensão e gravidez. Rev Bras Hipertens. 2002;9(3):256-61

8. Gomes MAA, Pierin AMG, Segre CA, Mion Junior D. Monitorização residencial da pressão arterial e monitorização ambulatorial da pressão arterial versus medida da pressão arterial no consultório. Arq Bras Cardiol. 1998;71(4):581-5.

http://dx.doi.org/10.1590/S0066-782X1998001000004

9. Penny JA1, Halligan AW, Shennan AH, Lambert PC, Jones DR, Swiet $\mathrm{M}$, et al. Automated, ambulatory, or conventional blood pressure measurement in pregnancy: which is the better predictor of severe hypertension? Am J Obstet Gynecol. 1998;178(3):521-6. http://dx.doi.org/10.1016/S0002-9378(98)70432-6

10. Alessi A. Uso de MAPA no tratamento anti-hipertensivo. Rev Bras Hipertens. 2006;13(3):203-7.

11. Pizzani L, Silva RC, Bello SF, Hayashi MCPI. A arte da pesquisa bibliográfica na busca do conhecimento. Rev Dig Bibl Ci Inf. 2012;10(1):53-66 
12. Prodanov CC, Freitas EC. Metodologia do trabalho científico: métodos e técnicas da pesquisa e do trabalho acadêmico. $2^{a}$ ed. Novo Hamburgo: Feevale; 2013.

13. Stergiou GS, Kollias A, Zeniodi M, Karpettas N, Ntineri A. Home blood pressure monitoring: primary role in hypertension management. Curr Hypertens Rep. 2014;16(8):462. http://dx.doi.org/10.1007/s11906-014-0462-8

14. O'Brien E, Parati G, Stergiou G. Ambulatory blood pressure measurement: what is the international consensus? Hypertension. 2013;62(6):988-94.

http://dx.doi.org/10.1161/HYPERTENSIONAHA. 113.02148

15. Marchioli M, Abbade JF, Peraçoli JC. Pressão arterial e frequência cardíaca avaliadas pela MAPA em primigestas durante o parto e puerpério imediato. Rev Bras Ginecol Obstet. 2004;26(5):391-8. http://dx.doi.org/10.1590/S0100-72032004000500008

16. Brown MA, Robinson A, Jones $M$. The white coat effect in hypertensive pregnancy: much ado about nothing? $\mathrm{Br} \mathrm{J}$ Obstet Gynaecol. 1999;106(5):474-80.

http://dx.doi.org/10.1111/j.1471-0528.2000.tb11595.x
17. Hermida RC, Ayala DE, Mojón A, Fernández JR. Time-qualified reference values for ambulatory blood pressure monitoring in pregnancy. Hypertension. 2001;38(3 Pt 2):746-52.

18. Ayala DE, Hermida RC. Ambulatory blood pressure monitoring for the early identification of hypertension in pregnancy. Chronobio Int. 2013;30(1-2):233-59.

http://dx.doi.org/10.3109/07420528.2012.714687

19. Pierin AMG, Mion JRD. Hipertensão, normotensão e o efeito do avental branco. In: Pierin AMG. Hipertensão arterial: uma proposta para o cuidar. Barueri: Manole; 2004. p.49-68.

20. Souza WKSB, Jardim PCBV, Porto LB, Araújo FA, Sousa ALL, Salgado CM. Comparação e correlação entre automedida, medida casual e monitorização ambulatorial da pressão arterial Arq Bras Cardiol. 2011;97(2):148-55.

http://dx.doi.org/10.1590/S0066-782X2011005000076 